\title{
Variables Significativas de Diseño Óptimo en Simuladores de Proceso
}

\author{
Omar A. Iglesias ${ }^{(1)}$, Juan D. Soto ${ }^{(1)}$ y Carmen N. Paniagua ${ }^{(1,2) *}$ \\ (1) Depto. de Ingeniería Química, Facultad de Ingeniería, Univ. Nac. de La Plata, 1 y 47, La Plata, \\ Argentina, (e-mail: oaiglesi@ing.unlp.edu.ar, incubadora@ing.unlp.edu.ar) \\ (2) Instituto de Investigación en Informática, LIDI, Facultad de Informática, Univ. Nac. de La Plata, \\ 1 y 47 , La Plata, Argentina, (e-mail: cnpaniag@ing.unlp.edu.ar) \\ * Autor a quien debe ser dirigida la correspondencia
}

Recibido Ago. 05, 2011; Aceptado Oct. 27, 2011; Versión Final recibida Dic. 07, 2011

\section{Resumen}

Se han desarrollado módulos para un simulador de procesos de código abierto, Sim42, accesible via Web. El objetivo es la consideración de las variables de diseño en la estructuración óptima de procesos industriales, en la enseñanza de la Ingeniería Química. El simulador debe ser capaz de a) estimar la inversión en equipos, costos de servicios e insumos e ingresos por venta y b) manejar un método de optimización para problemas no lineales restringidos. Como estas facilidades no existían en Sim42, se agregaron nuevos módulos al código disponible, así como otros que completan el análisis. Los desarrollos se aplican a la simulación, análisis y optimización de un ciclo Linde para licuar una corriente de metano. Se concluye sobre la conveniencia de contar con un simulador de código abierto que permita adecuar sus prestaciones a los requerimientos de los conceptos a transmitir.

\section{Significant Variables of Optimum Design in Process Simulators}

\begin{abstract}
Some modules have been developed for an open code process simulator, Sim42, accessible through the Web. The objective is the consideration of the design variables in the optimum structuring of industrial processes, in the teaching of Chemical Engineering. The simulator must be able to: a) estimating the investment in equipments, the costs of services and inputs and the revenues for sale and b) managing an optimization method for non lineal restricted problems. Since both facilities do not exist in Sim42 new modules were added to the available code, as well as others ones that complete the analysis. The developments are applied to the simulation, analysis and optimization of a Linde cycle to liquefy a methane stream. It is conclude that having an open-source simulator that allows adapting its performance to the requirements of the concepts to be conveyed is highly convenient.
\end{abstract}

Keywords: simulation, optimization, non lineal programming, costs estimate, significant variables 


\section{INTRODUCCIÓN}

La concreción de las etapas finales del diseño de unidades de Ingeniería Química requiere utilizar simuladores de procesos para la evaluación de alternativas y la determinación de las condiciones óptimas de operación. La enseñanza de alguno de estos temas exige una consideración criteriosa de ciertos aspectos prácticos, que exceden el exclusivo enfoque matemático [Iglesias y Paniagua, 2006]. Así, los grados de libertad de un determinado problema de diseño no son, necesariamente, el número de variables de decisión que, en la práctica, han de usarse en la búsqueda del óptimo. A través de un análisis del problema se puede concluir cuales son las variables más convenientes para consumir, fijando su valor, los grados de libertad que presenta el mismo. Pero la incidencia de ellas sobre la función objetivo que se haya adoptado no es igual en todos los casos. Esto plantea una primera fase en el proceso de optimización, que consiste en determinar la significación de las variables independientes, es decir, la sensibilidad de la función objetivo a las mismas. El tema, por su incidencia práctica sobre la complejidad del cálculo en la búsqueda del óptimo, ha despertado la atención de numerosos investigadores en las más diversas disciplinas, con el uso de un amplio abanico de técnicas diferentes para abordar el problema, desde enfoques heurísticos, como el propuesto en los 80 por el Diseño Conceptual (Douglas, 1988) hasta el tratamiento mediante herramientas estadísticas (por ejemplo, Hasan et al., 2005; Lu y Mei, 2007).

Es evidente la necesidad de un algoritmo de optimización para problemas no lineales restringidos así como de funciones para la estimación de inversiones y costos variables. Los principales simuladores comerciales tienen incorporado un método de optimización acoplable a la simulación. Esto es crucial ya que, si bien los programas admiten el agregado de nuevos módulos, remediar la falta de un optimizador resulta difícil desde el punto de vista informático, con independencia de la complejidad matemática del algoritmo de optimización que se elija. No ocurre lo mismo con las funciones de estimación económica para equipos y corrientes de proceso, donde no existe mayor dificultad, toda vez que se encuentren disponibles las dimensiones de los equipos.

El agregado de una rutina de análisis automático de significación presenta similares dificultades informáticas. Ningún simulador de procesos, comercial o no, permite realizar ese análisis. Cualquiera sea la técnica que se adopte para hacerlo, siempre habrá que simular el sistema, primero, para un caso base, asignando valores aconsejados por la práctica (Branan, 2005) a las variables de diseño que agotan los grados de libertad que tiene el problema. Luego, ese caso base será perturbado, en un proceso que dependerá de la metodología que se adopte. A la luz de los resultados encontrados se ha de estimar la sensibilidad de la función objetivo a las distintas variables de diseño y, en consecuencia, establecer cuales resultan significativas para el problema. Será sobre este subconjunto que se efectuará la optimización, conservando el resto el valor típico adoptado. Aunque engorrosa, la operatoria que conduce a la determinación de las variables significativas es abordable manualmente por el usuario, utilizando el simulador y llevando registro de los resultados obtenidos en los sucesivos pasos.

La enseñanza de estos temas plantea una dificultad adicional cuando se adoptan estrategias no presenciales en cursos de actualización profesional, ya que ninguno de los productos comerciales, como ASPEN Plus $($, ASPEN Hysys $(\odot)$ o PRO/IIC, son accesibles en forma remota, lo que limita seriamente las posibilidades de realización de tareas fuera del horario laboral. Los autores debieron abordar todas estas cuestiones como consecuencia de los requerimientos que planteaban los cursos sobre temas de Ingeniería de Procesos. Debía contarse, para posibilitar un trabajo uniforme de los alumnos, con un simulador accesible en forma remota. Este debía admitir el agregado de nuevos módulos para cubrir las falencias que se detectasen al tratar de aplicarlo en los distintos temas de los cursos. La búsqueda concluyó por seleccionar el simulador de procesos de código abierto Sim42 (Cota et al., 2003, Mohajer et al. 2008, Dias et al., 2009), desarrollado en la Universidad de Calgary, Canadá.

\section{EL SIMULADOR SIM42. MODIFICACIONES INTRODUCIDAS}

Sim42 está bajo licencia de software libre BSD que permite la libre distribución y modificación de la totalidad del código fuente sin exigir la redistribución de las modificaciones realizadas. El 
simulador está escrito en Python (http://www.python.org), un lenguaje de programación multiplataforma de alto nivel orientado a objetos, claro y muy potente. Los autores del núcleo original buscaron independizarlo de las interfaces de usuario y la provisión de los métodos termodinámicos, siendo posible incorporar desarrollos propios para cualquiera de los casos. La versión disponible de Sim42 incluye SimBA, una interfase que utiliza un navegador Web como Internet Explorer@ o Firefox@, lo que habilita su acceso remoto.

En lo que a este trabajo se refiere, la estructura básica del simulador es la que se muestra en la figura 1. El Command Interface es, por una parte, la interfase de comunicación con el núcleo (kernel) del sistema operativo y, por otra, está a cargo de direccionar los requerimientos del usuario hacia Flowsheet, el supervisor del desarrollo (ordenamiento del cálculo, resolución de reciclos, etc.) de la simulación, cuyos cálculos se llevan a cabo en las distintas unidades (UnitOp) que representan las operaciones que componen el sistema simulado.

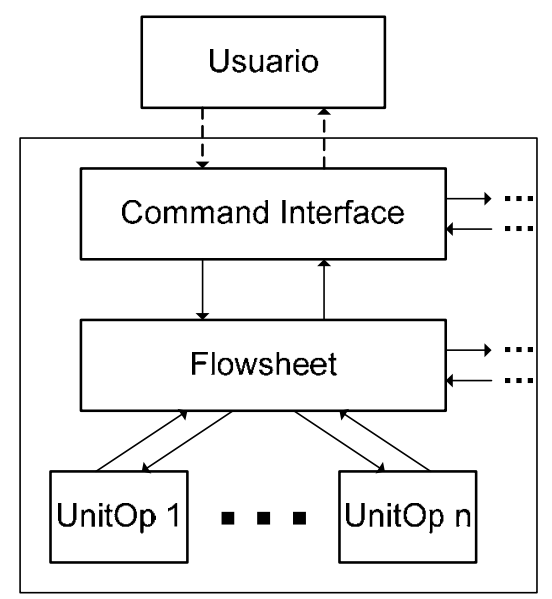

Fig. 1: Estructura básica de Sim42

Una característica importante de Flowsheet es su capacidad para propagar la información parcial generada, hacia atrás y adelante del punto donde se produce, lo que permite solucionar muchos problemas complicados sin necesidad de cálculos iterativos.

Adicionalmente, Command Interface y Flowsheet se comunican con el núcleo de administración de propiedades termodinámicas, Thermo Admin, pero dichos vínculos carecen de interés en lo que a este trabajo se refiere. Esta estructura es, en esencia, análoga a la de cualquier simulador modular de procesos y, por ende, la naturaleza de las dificultades encontradas en los desarrollos realizados también estarán presentes, en esencia, en cualquier otro simulador.

En la versión disponible del simulador no existía capacidad para estimar inversiones o costos variables ni había un algoritmo para la optimización de problemas no lineales restringidos. Obviamente, no se contaba con facilidades para realizar un análisis de significación de variables de diseño. Todo esto debería ser incorporado como desarrollos adicionales, aprovechando la naturaleza abierta del código de Sim42, otra característica determinante en la selección realizada.

En la estructura básica de la figura 1 se puede ver que la estimación de costos y amortizaciones puede realizarse a nivel de cada unidad o, eventualmente, estructurar, en una unidad separada, todos los cálculos necesarios para los diferentes tipos de equipos y corrientes de insumos o productos y que sea esta unidad la que, a requerimiento del resto, transfiera el valor calculado.

La incorporación de módulos para realizar el análisis de significación o la búsqueda de un óptimo ya no resulta tan simple. En ambos casos el sistema debe simularse reiteradamente, modificando los valores asignados a una o más de las variables de diseño, antes de que la operación esté en condiciones de brindar un resultado. En el caso del análisis de significación, el número en que se debe repetir la simulación está determinado por la metodología adoptada. Para la optimización, el mismo dependerá de las condiciones de partida y del valor de la cota final que se fije. 
En ambas situaciones, la nueva unidad deberá comportarse como un usuario que modifica los valores de las variables de decisión, recoge los resultados de esa modificación, considera la condición de final que corresponde al tipo de análisis en curso y, en función de esto, decide continuar con las simulaciones o dar por terminado el estudio, habilitando la exposición de las conclusiones obtenidas. Lo anterior plantea, con claridad, que el accionar de estos nuevos módulos debe ubicarse a nivel del propio Command Interface, tomando el control de la simulación mientras se desarrolla el proceso de estudio planteado y devolviéndolo cuando éste ha concluido.

En las modificaciones introducidas a Sim42, el agregado de elementos económicos al simulador se llevó a cabo sobre los módulos de los equipos y corrientes de materia y energía disponibles. En los primeros fue preciso agregar, a veces, rutinas de diseño para contar con las dimensiones físicas de los equipos y poder estimar, a partir de ellas, el monto de la inversión. Este estimado se realiza de acuerdo a los esquemas clásicos utilizados en la industria de procesos. En particular, para los agregados en Sim42 se eligieron las modificaciones de las correlaciones de Ulrich del texto de Turton et al.(1998). En esto se tuvo en cuenta que esa fuente era la más moderna a la que se podía acceder con información coherente para los distintos equipos. Como en todos los casos de este tipo de estimaciones, los valores correlacionados corresponden a un determinado tiempo, 1996 en este caso, y para la actualización de los mismos se utiliza el Chemical Engineering's Plant Cost Index (CEPCI), publicado en la revista Chemical Engineering.

Para las otras modificaciones se planteó la conveniencia de generar dos módulos nuevos, de modo de habilitar futuros agregados de otras metodologías de análisis de significación y algoritmos de búsqueda de óptimos. Como quedó expresado anteriormente, en el desarrollo de esos módulos, además de generar el código con las operaciones propias de las metodologías seleccionadas, fue necesario programar, a nivel de Command Interface, las interrupciones del flujo normal de la simulación, para permitir la resolución del sistema con los distintos conjuntos de valores de las variables de diseño bajo análisis, antes de dar por terminado el proceso.

En una primera instancia, se eligió, por la simplicidad de los esquemas de cálculo, realizar el análisis de significación de variables de diseño con el esquema propuesto por Douglas en su ya clásico Diseño Conceptual y para la búsqueda de óptimos, el algoritmo Complex (Edgar y Himmelblau, 2001), un método politópico que no exige el cálculo de derivadas. Para el análisis de significación, el estudio de sensibilidad se realiza a partir de la estimación de las derivadas parciales de las componentes de la función objetivo respecto de cada variable de diseño, valores conseguidos mediante perturbaciones controladas del sistema simulado. Para ello, tras la resolución del caso base, deben realizarse, como mínimo, un número de simulaciones igual al de las variables de diseño existentes, modificando, cada vez, el valor de una sola de esas variables.

En lo que respecta a la implementación del Complex se debió tener en cuenta que, al igual que todo algoritmo de optimización de problemas con restricciones, el sistema en consideración debe informar de las violaciones a las fronteras impuestas, para proceder en consecuencia. En el caso de una planta de proceso, existen, en el funcionamiento de los equipos que la componen, límites que imponen las leyes de la física y de la química. En el desarrollo de los módulos que permiten simular los equipos se incluyen estas limitaciones, cuya trasgresión, fatal para el curso de la simulación, da origen a mensajes de error. De este modo, el propio simulador evalúa el cumplimiento de las restricciones fisicoquímicas o termodinámicas que tiene el problema. Para usarlo como tal, cada vez que se proponga un conjunto de valores para las variables de diseño, habrá que verificar que no se produzcan mensajes de error a nivel de las unidades vinculadas a Flowsheet y, en caso de que ocurran, evitar que Command Interface detenga la simulación, para poder realizar una nueva propuesta de las variables de diseño.

En el sistema simulado pueden existir, además, restricciones técnicas, cuya violación no es detectada en los módulos, ya que la limitación es del problema, no del funcionamiento de los equipos. Todas estas restricciones, técnicas o no, pueden afectar tanto a las variables de diseño como a las de estado, que se calculan a partir de ellas. Para poder tener en cuenta los límites técnicos del problema se agregó un módulo donde el usuario ingresa las relaciones en forma de ecuaciones, haciendo uso de variables flojas, $g(\underline{X}) \leq 0 \rightarrow g(\underline{X})+S=0$ y la restricción se cumple si 
$\mathrm{S}$ es no negativo. Aquí se ingresan dos grupos de restricciones, uno, que afecta exclusivamente a las variables de decisión y otro a la totalidad de las variables. Esta división permite proceder a la simulación sólo sobre soluciones que, en principio, son factibles, con el consiguiente ahorro de tiempo. La secuencia del análisis de restricciones que el Complex realiza durante el proceso de optimización consta de tres pasos: primero, verifica las restricciones técnicas que afectan a las variables de diseño para evitar la simulación de una solución no factible; si se cumplen, procede a simular el sistema; si no hay error, verifica las restricciones técnicas donde participan todas las variables y si se satisfacen, el punto propuesto es factible, de lo contrario, debe proponerse otro.

\section{EJEMPLO DE APLICACIÓN}

En la figura 2 se muestra el esquema de proceso simplificado de una planta de licuación de $\mathrm{CH}_{4}$ que usa un ciclo Linde con 3 etapas de compresión.

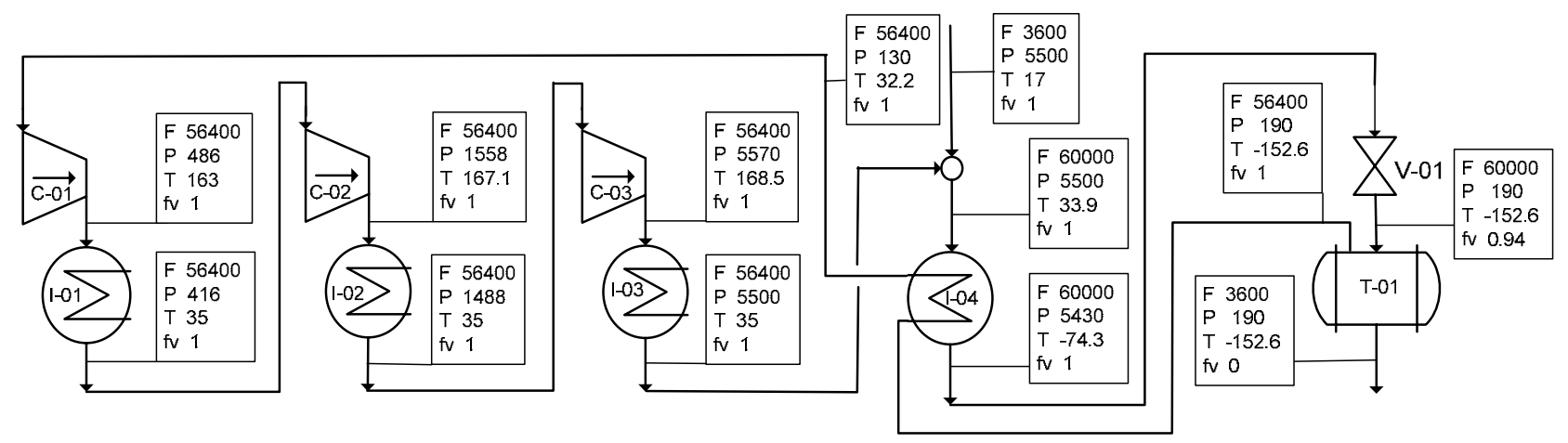

Fig. 2: Planta de licuación de $\mathrm{CH} 4$ (Ciclo Linde)

Se trata de licuar un caudal $\mathrm{F}$ de $3600 \mathrm{Kg} / \mathrm{h}$ de $\mathrm{CH}_{4}$ gaseoso $(\mathrm{fv}=1)$, disponible a una presión $\mathrm{P}$ de $5500 \mathrm{kPa}$ y una temperatura $\mathrm{T}$ de $17^{\circ} \mathrm{C}$. La corriente gaseosa se expande en la válvula $\mathrm{V}-01 \mathrm{y}$ la fracción condensada se separa en el tanque T-01. El $\mathrm{CH}_{4}$ no condensado se recomprime y se lo mezcla con la alimentación. Para los enfriamientos post compresión se dispone de $\mathrm{H}_{2} \mathrm{O}$ a $25^{\circ} \mathrm{C}$. Las pérdidas de carga en los equipos de intercambio se estiman iguales a $70 \mathrm{kPa}$ por lado.

Como se ha dicho (Iglesias y Paniagua, 2006) toda simulación debe comenzar por un análisis de los grados de libertad del problema que, en este caso, resultan ser 7. Para consumirlos, una elección adecuada de las variables conduce a elegir la presión y la fracción vaporizada después de V-01, los valores de $\mathrm{P}$ a la salida de los compresores C-01 y C-02 y las temperaturas del $\mathrm{CH}_{4}$ que deja I-01, I-02 y I-03. El caso base se genera al darle a estas variables valores razonables. En la figura 2 aparecen indicados los resultados encontrados en la simulación de ese caso para el caudal, la presión, la temperatura y la fracción vaporizada en distintos puntos de la planta. En este momento se está en condiciones de efectuar el análisis de significación de las variables de diseño elegidas. En rigor, esto podría obviarse, ya que una consideración conceptual del caso permite detectar cuales variables inciden significativamente sobre el Costo Total Anual (CTA), la función objetivo propuesta. El sector de compresión es el responsable de las mayores erogaciones, tanto por la inversión que requiere como por el gasto en energía eléctrica. Ambos ítems estarán influenciados por dos factores: el salto total de presión y el caudal de gas que procesa el sector. Es esperable que las variables significativas sean los valores de $P$ y fv a la salida de V-01. Al hacer el análisis con el módulo desarrollado se encuentra este resultado.

Adicionalmente, se realizaron algunas corridas del módulo de análisis, modificando, arbitrariamente, los rangos de variación admitidos para las distintas variables. Pudo detectarse algún caso en el que los parámetros de significación presentaban variaciones importantes. Esto plantea, por una parte, la necesidad de abordar este tipo de estudios con conocimientos sólidos sobre el problema y, por otra, la conveniencia de analizar otras metodologías que resulten menos sensibles a desconocimientos parciales del diseñador. 
Luego de completado el análisis de significación se puede comenzar con la búsqueda de condiciones óptimas. Con fines comparativos se realizó la optimización, primero, considerando las 7 variables de diseño existentes y, luego, sólo las significativas, dejando a las restantes en los valores iniciales adoptados. Se encuentra que el valor de la función objetivo se incrementa, en este último caso, en menos del $0,4 \%$ en tanto que en los valores de las variables de diseño se verifican variaciones menores al $1 \%$. En la primera parte se pudo apreciar que, aún cuando las variables no significativas modifican sustancialmente su valor inicial, la incidencia sobre el CTA es, como era de esperar, casi irrelevante. Finalmente, las distintas resoluciones realizadas permiten aseverar que la implementación en Sim42 del método Complex, con la estructura planteada anteriormente, tiene un comportamiento totalmente satisfactorio en el proceso de optimización de la planta de licuación.

\section{CONCLUSIONES}

El trabajo realizado pone de manifiesto la conveniencia del software de código abierto en los desarrollos para la enseñanza de la Ingeniería. En particular, cuando el programa original ha sido concebido para su utilización vía Web, se habilita la posibilidad de incorporarlo a cursos de actualización, orientados a graduados con trayectorias profesionales.

Queda claro que el análisis de significación de variables de diseño es una potente herramienta en la optimización de procesos. Sin embargo, requiere una experiencia importante del diseñador en el sistema simulado. De lo contrario, el análisis puede volverse sensible a la incorrección de la información suministrada, cuestión que pudo verificarse sobre la metodología elegida para el desarrollo. En este sentido, se ha de continuar con esta línea de trabajo, analizando otros enfoques para realizar el estudio de sensibilidad.

\section{REFERENCIAS}

Branan, C.R., Rules of Thumb for Chemical Engineers, 4ª ed., Gulf Professional Publishing (2005).

Cota, R., M. Satyro, M. Craig, B. Svrcek y B.Young, Development of an Open Source Chemical Process Simulator, Modeling \& Simulation, 525-530 (2003).

Dias R.S., L. Cardoso Silva y A.J. de Assis, Plant wide simulation using the free chemical process simulator Sim42: Natural gas separation and reforming, Computer Applications in Engineering Education (on line) (2009).

Douglas J-M., Conceptual Design of Chemical Processes, McGraw Hill Inc. (1988)

Edgar T.F. y D.M. Himmelblau, Optimization of Chemical Processes, $2^{\mathrm{a}}$ ed., McGraw-Hill (2001).

Hasan, S.D.M., D.N.C. Melo y R.M. Filho, Simulation and response surface analysis for the optimization of a three-phase catalytic slurry reactor, Chemical Engineering and Processing, 4(3), 335-343 (2005).

Iglesias O.A. y C.N. Paniagua; Using Online Simulation in Teaching Alternative Analysis and Process Optimization, Current Developments in Technology-Assisted Education - FORMATEX, 2075-2080 (2006).

Lu Y. y L. Mei; Optimization of fermentation conditions for P450 BM-3 monooxygenase production by hybrid design methodology, J Zhejiang Univ Sci B, 8(1), 27-32 (2007).

Mohajer M., B.R. Young y W. Svrcek, Comparing Pressure Flow Solvers for Dynamic Process Simulation, Chemical Product and Process Modeling, 3(1), Art.13, (2008).

Turton R., R. Bailie, W. Whiting y J. Shaeiwitz, Analysis, Synthesis, and Design of Chemical Processes. Prentice Hall PTR (1998). 